\title{
A RELATIONAL DISTANCE-BASED FRAMEWORK FOR HIERARCHICAL IMAGE UNDERSTANDING
}

\author{
Laura Antanas ${ }^{1}$, Martijn van Otterlo ${ }^{1}$, José Oramas M. ${ }^{1}$, Tinne Tuytelaars ${ }^{1}$ and Luc De Raedt ${ }^{1}$ \\ ${ }^{1}$ Katholieke Universiteit Leuven, Belgium
}

Keywords: hierarchical image understanding, relational instance-based learning, structured representations

\begin{abstract}
Understanding images in terms of hierarchical and logical structures is crucial for many semantic tasks, including image retrieval, scene understanding and robotic vision. This paper combines compositional hierarchies, qualitative spatial relations, relational instance-based learning and robust feature extraction in one framework. For each layer in the hierarchy, substructures in the images are detected, classified and then employed one layer up the hierarchy to obtain higher-level semantic structures, by making use of qualitative spatial relations. The approach is applied to street view images. We employ a four-layer hierarchy in which subsequently corners, windows and doors, and individual houses are detected.
\end{abstract}

\section{INTRODUCTION}

Interpreting visual scenes is a hard task and the field of computer vision has developed many techniques over the past decades for segmentation, classification, recognition and retrieval of images, objects and scenes, e.g., (Li et al., 2009; Sudderth et al., 2008). Many of those techniques use a plethora of local low- to medium-level features such as geometric primitives, patches, point clouds and invariant features (Tuytelaars and Mikolajczyk, 2007). However, for high-level tasks such features are potentially not enough. It is more intuitive to understand and describe visual scenes in terms of hierarchical structural or graph-like representations, which express the natural composition of scenes into objects, parts of objects and lower-level substructures (Pinz et al., 2009). Man-made (vs. natural) scenes especially exhibit considerable structure that can be captured using qualitative spatial relations. For example, a typical house consists of aligned elements such as: a roof, some windows, one or more doors and possibly a chimney. A hierarchical aspect is that a window and a chimney themselves are composed of particular configurations of local features (e.g., corners with a certain appearance arranged in a rectangular-like way and 'brick'like patterns of a certain shape, respectively).

This view on hierarchical image representation builds on very early ideas that hierarchical structure and relational constraints are key components of an image understanding system (Hanson and Riseman, 1978). However, then, different from today, lowand mid-level vision procedures were too immature to support such ambitious representations and goals. In this paper we renew the idea that visual scenes are best described using high-level representational devices such as graphs, and even more generally using logical languages (De Raedt, 2008). The clear advantage of these rich symbolic representations is that they can, for example, abstract spatial relations between scene components away from exact locations and generalize over similar situations, independent of the metric details. In this paper we describe a novel, model-free relational distance-based technique for hierarchical image understanding. It considers the structural aspect of a scene and in this scope it employs recent developments from relational learning. Instead of using a formal model of the distribution of scenes (e.g., in the form of a grammar), we start from a set of annotated examples of objects in the scene. Yet, our framework preserves desired properties of grammars, that is, it employs structured input features and outputs a structured explanation of the image at each layer in the hierarchy. The base layer relies on local feature descriptors. A subsequent layer consists of objects and higher levels consist of configurations of objects. Spatial logical representations are used to generalize over configurations with different number of components. We explicitly focus on the recognition of known substructures in street view images (i.e., windows, doors and houses), however, our approach can be used for other domains as well.

Our main contribution is a new framework in which spatial configurations and relational distance functions are used throughout all levels of a hierarchy, in a unified way, to recognize known objects. Many 
computer vision algorithms use probabilistic classifiers, distance functions and kernels for object detection. Yet, these techniques are less well equipped for detecting higher-level concepts that consist of qualitative spatial configurations of objects, for which relational generalization techniques (De Raedt, 2008) are required. Thus far, most work in computer vision has focused on fixed compositional structures (Felzenszwalb et al., 2010) or constellation models (Fergus et al., 2007). We further show how recent results in relational distance metrics (De Raedt and Ramon, 2009) can be utilized as a generalization technique to help recognize higher-level structures in an image. We assume manually labeled examples of object categories we want to recognize throughout all layers in the hierarchy (i.e., houses, windows and doors). Each house is annotated with the locations and shapes of its constituent windows and doors. We represent an object as a set of parts and a set of qualitative spatial relations defined on them (hence; a relational attribute graph). Each image substructure is spatially embedded in a 2D plane, and parts are related to each other with respect to this space. A strong point of our framework is that distance functions at each level of the hierarchy, either in terms of low-level features or high-level relational spatial composites, can easily be replaced by alternatives. In addition to a novel framework combining robust visual features and relational generalization techniques, we introduce a new annotated dataset derived from Google StreetView Images.

\section{RELATED WORK}

Several papers have applied computer vision techniques to house facades domain. In (Hartz and Neumann, 2007; Hartz, 2009) structure models of meaningful facade concepts are learned from examples. In (Zhao et al., 2010) the authors tackle the house delineation problem by generating vertical separating lines on the facade and using a dissimilarity measure between these features. Finally (Müller et al., 2007; Koutsourakis et al., 2009) assume to have the structure of a building facade and then estimate the parameters of the model. Different from these, our work uses distances between logical interpretations to detect known structures in an instance-based fashion.

In other domains, i.e., document analysis, distance-based techniques have been used in a relational setting (Esposito et al., 1992), yet they do not address the intrinsically noisy nature of visionbased interpretation of images of houses. In fact, most papers that do address such problems use a modelbased approach and perform interpretation through

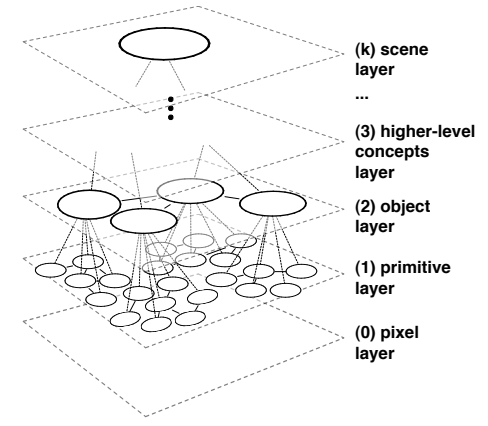

Figure 1: A typical hierarchy with $k+1$ layers. A layer $i$ is a set of classified entities (empty circles) arranged in spatial configurations. Each configuration generates a classified entity at the next higher-level $i+1$ in the hierarchy.

image grammars (Hartz and Neumann, 2007; Lippow et al., 2008; Felzenszwalb et al., 2010). These have been well-studied in the literature (Zhu and Mumford, 2006), but need considerably more bias (or learning procedures) to supply (or learn) the grammar rules. This in contrast to our model-free approach, which is based on comparison to annotated examples. The use of rich logical formalisms in non-grammar approaches by the state-of-the-art in computer vision is limited (Szeliski, 2010). Closely related are graph matching and graph kernel-based techniques for image understanding (Caetano et al., 2009; Harchaoui and Bach, 2007). However, different from these, our framework builds on recent general results on distance metrics for logical interpretations (De Raedt and Ramon, 2009). In this sense, we approach a current interest in using relational learning techniques for complex vision tasks (Petrou, 2008). Other relevant work includes approaches based on relational object models (Bar-Hillel and Weinshall, 2008) or probabilistic relational learning (Dubba et al., 2010).

\section{HIERARCHICAL IMAGE UNDERSTANDING}

In our hierarchical framework an image $Z$ is described at several layers $0, \ldots, k$ in the hierarchy, with 0 the base layer and $k$ the top layer (Figure 1). At each layer, the description consists of a set of classified regions of interest (or parts) $C_{i}$ as well as the spatial relationships among them. The classes denote the concepts the parts belong to. The task then consists of using the description of an image at level $i$ to obtain and classify the parts $C_{i+1}$ at the next higher level $i+1$ in the hierarchy. We call this the delineation (or semantic segmentation) task. We assume that annotated images at all layers are available as training data. 
In our case, the base layer consists of the image itself, while the parts are pixels. In the primitive layer the parts are local patterns, e.g., a corner. The object layer is then built from spatial configurations of such local patterns, forming regions of interest belonging to concepts such as door and window. These are then used at the next level to find higher-level regions of interest representing houses. We stop at the scene layer which groups houses into streets. Each layer consists of parts and the classes they belong to, and it is formed by making use of spatial configurations of parts from the previous lower-level layer. This hierarchical image understanding framework propagates the detected parts in a bottom up manner through each layer. Information flow is similar at all levels; first, the parts $C_{i-1}$ of the previous layer are detected, then current-level parts are generated using configurations of $C_{i-1}$ and finally the best ones $C_{i}$ are kept to be further employed at the next layer.

\section{REPRESENTATION AND FUNCTION OF ONE LAYER}

Let us describe in more detail how to represent an image $Z$ at one layer in the hierarchy. We assume knowledge about the identity of the layer and access to automatically detected and extracted regions of interest in the image at this layer, together with their descriptors. Based on these assumptions we define a language consisting of visual entities, spatial relations between visual entities, composite entities and membership relations between a visual entity and a composite entity. The language can differ from one layer to another, depending on the characteristics of the parts (or entities) at each layer.

A visual entity (vent $(\mathrm{id}, \operatorname{attr} 1, \ldots))$ represents an entity of the image at the current layer $i$, e.g., a corner or a window with id as its unique object identifier. Attributes of a visual entity are its position, i.e., the coordinates of the visual entity bounding box, and a class or concept label. Spatial relations impose a structure on visual entities (e.g. spatial neighborhood) and are defined using a logical background theory (a set of Prolog rules as in relational learning). As one example consider the spatial relation cRight $\left(i d_{1}, i d_{2}\right.$, dist) (close aligned horizontally to the right) with an attribute for the Euclidean distance dist between two visual entities $i d_{1}$ and $i d_{2}$. A composite entity (cent $(\mathrm{ic}, \operatorname{attr} 1, \ldots))$ is a candidate visual entity at the next level $i+1$; it consists of a set of visual entities at level $i$ and the relations that hold among them; thus it implicitly groups a set of visual entities into a composite entity, using the part-of re- lation. Thus, composite and visual entities are linked through membership relations partof(id,ic). All visual entities, composite entities, spatial and membership relations for image $Z$ at one layer are denoted $V_{Z}, C_{Z}, S_{Z}$ and $M_{Z}$, respectively. We define a visual interpretation $I_{Z}$ of an image $Z$ as their union. For any composite entity $c$ we denote $V_{c}$ as the set of visual entities grouped by $c, S_{c}$ as the set of spatial relations representing the projection of $S_{Z}$ on $V_{c}$ and $M_{c}$ as the set of membership relationships between the elements of $V_{c}$ and $c$ itself. We further denote $I_{c}$ as the subset of $I_{Z}$ that contains the visual entities $V_{c}$, their spatial relations $S_{c}$ grouped by $c$, corresponding membership relations $M_{c}$ and $c$ itself. $V S_{c}$ finally consists of $V_{c}$ and $S_{c}$ in $I_{c}$, while $V S_{Z}$ is determined by $V_{Z}$ and $S_{Z}$ in $I_{Z}$. An example of a visual interpretation at the house layer for Figure 2(a) is given in Figure 2(c). Some elements of $C_{Z}$ capture the inherent structure of the concept house; the rest belong to the category none.

It is convenient to visualize interpretations as graphs in which the entities correspond to vertices and the relations to directed (labeled) edges. A composite entity then denotes the subgraph $V S_{c}$. The task is to construct the set of relevant composite entities $C$ such that the visual entities for level $i+1$ can be obtained. This is a combinatorial problem as potentially all subgraphs in $V S_{Z}$ are candidates. The generation of meaningful new entities is also a novel task in the relational learning context. It can be seen as a dual to predicate invention (Muggleton and Buntine, 1988). There the goal is to determine new and useful predicates. Here the task is to invent new entities. In a probabilistic context, it is related to existence uncertainty, a term coined in the literature on probabilistic relational models (Getoor et al., 2000).

Our goal is to recognize known visual structures in a new image. We approach it in two main steps: classification and selection. In the classification step, for each of the candidate visual entities in $C$, we need to determine the concept they belong to (if any). This can be cast into a concept-learning problem. For each target class (such as house, window and door) we have examples in our training set. Consider, for instance, the concept of a house. In Figure 2(b) the composite entity $i c_{1}$ forms a positive example, while $i c_{2}$ is a negative example. For each composite entity $c$ that forms a positive example of a concept, we use the visual interpretation $V S_{c}$ as prototype. Such a prototype is shown as a graph in Figure 4 on the left, where it is matched with a part of another image interpretation. Its corresponding visual interpretation is presented in Figure 3. The composite entity classification task is solved using an instance based learning approach. We use a distance measure to find the best matches of can- 


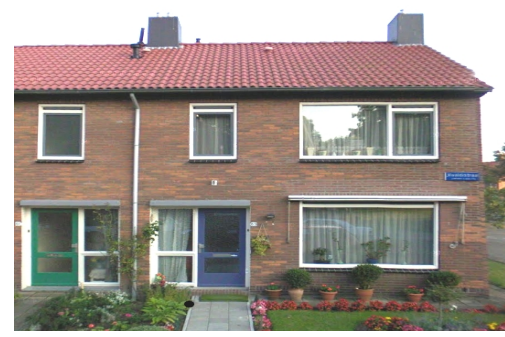

(a) An image $Z$.

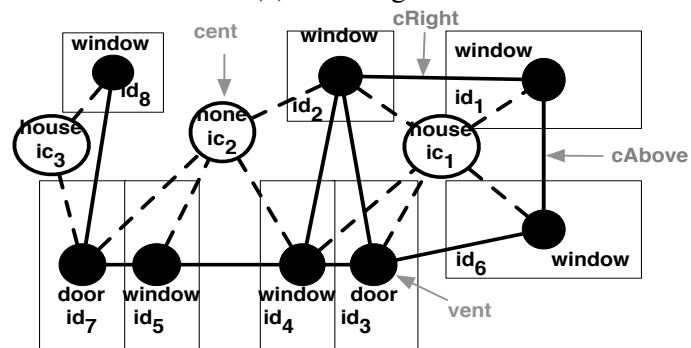

(b) Graphical representation of image $Z$. Each visual entity corresponds to a detected door/window (black circles in white bounding boxes describing spatial locations $l_{i}$ ) and each composite entity is a possible house defined by a subgroup of these objects (white circles). The spatial and membership relations are marked by the continuous and interrupted lines, respectively.

$I_{Z}=\left\{\operatorname{vent}\left(i d_{1}, l_{1}\right.\right.$, win $), \operatorname{vent}\left(i d_{2}, l_{2}\right.$, win $)$, $\operatorname{vent}\left(i d_{3}, l_{3}\right.$, door $)$, vent $\left(i d_{4}, l_{4}\right.$, win $)$, $\operatorname{vent}\left(i d_{5}, l_{5}\right.$, win $), \operatorname{vent}\left(i d_{6}, l_{6}\right.$, win $)$, $\operatorname{vent}\left(i d_{7}, l_{7}\right.$, door $)$, vent $\left(i d_{8}, l_{8}\right.$, win $)$, cRight $\left(i d_{1}, i d_{2}, d_{1}\right)$, cAbove $\left(i d_{2}, i d_{3}, d_{2}\right)$, cRight $\left(i d_{3}, i d_{4}, d_{3}\right), \operatorname{cRight}\left(i d_{4}, i d_{5}, d_{4}\right), \ldots$, cent $\left(i c_{1}, l_{9}\right.$, house $), \operatorname{cent}\left(i c_{2}, l_{10}\right.$, none $)$ cent $\left(i c_{3}, l_{11}\right.$, house $), \operatorname{part0f}\left(i d 1, i c_{1}\right), \operatorname{part0f}\left(i d_{2}, i c_{1}\right)$, part0f $\left.\left(i d_{2}, i c_{2}\right), \operatorname{part0f}\left(i d_{5}, i c_{2}\right), \ldots\right\}$.

(c) Corresponding visual interpretation $I_{Z}$ at the house layer for image $Z$. Visual entities are denoted with vent while composite entities with cent. Spatial relations are cRight (close to the right) and cAbove (close above). The membership relation is denoted part0f.

Figure 2: Image representation at one layer.

didate composite entities with prototypes.

The entity classification step classifies each entity in $C$ in a local manner, that is, this step only takes into account the entity to be classified and the set of prototypes, but no context. This may give unintuitive results at the global level. For instance, it could be that two entities with a significant overlap are both classified as houses. Therefore, we also perform a selection step in which contextual constraints are taken into account. Using global optimization we find the best subset $C^{*}$ of the classified entities in $C$. From $C^{*}$ we then derive detections.

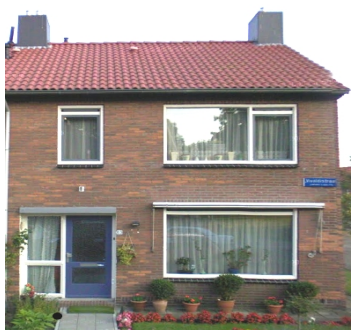

$V S_{c}=\left\{\operatorname{cent}\left(i c_{1}\right.\right.$, house $)$,

$\operatorname{vent}(i d 1, l 1$, win $), \operatorname{vent}(i d 2, l 2$, door $), \operatorname{vent}(i d 3, l 3$, win $)$, $\operatorname{vent}(i d 4, l 4$, win $)$, vent $(i d 6, l 6$, win $)$, cRight $(i d 1, i d 2, d 1)$, cAbove(id2,id3, d2), cRight(id3,id4, d3), cAbove (id 2,id4, d4), cRight (id6,id3, d5), cAbove $(i d 1, i d 6, d 6)$, part0f $\left(i d_{1}, i c_{1}\right)$, part0f $\left(i d_{2}, i c_{1}\right)$, part0f $\left.\left(i d_{3}, i c_{1}\right), \operatorname{part0f}\left(i d_{4}, i c_{1}\right), \operatorname{part0f}\left(i d_{6}, i c_{1}\right)\right\}$.

Figure 3: An example of an instance in the house facade domain at the house layer. The target attribute is the category of the composite entity, i.e., house in this case.

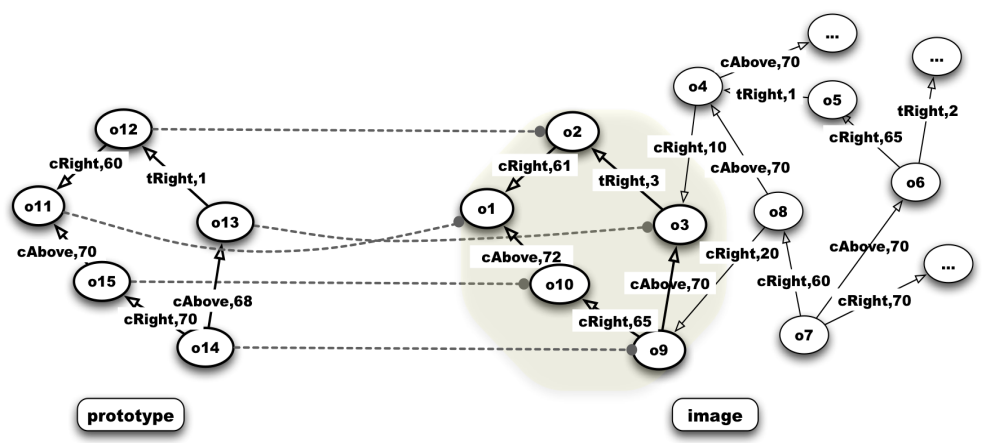

Figure 4: Graph representations of a prototype (left) and an image interpretation (right).

\section{LAYER-WISE SEMANTIC SEGMENTATION}

Next, we explain how composite generation, classification and selection functions work and fit together to obtain the delineation at one layer in the hierarchy. Intuitively, given an image $Z$ we detect known structures by trying to best embed prototypes in $Z$. To this end, we first generate composite entities, we then classify and select them, and finally we obtain class detections (Algorithm 1).

I Composite Entity Generation (GENERATE). We generate the set of relevant composite entities $C$ for an image $Z$ using a language bias, common in relational learning. As the number of all composite entities $C_{Z}$ is exponentially large in the size of $V_{Z}$, we impose an upper bound on the number of composite entities considered. The bound, calculated image-wise, is proportional to the size of $V_{Z}$, but not larger than a heuristi- 


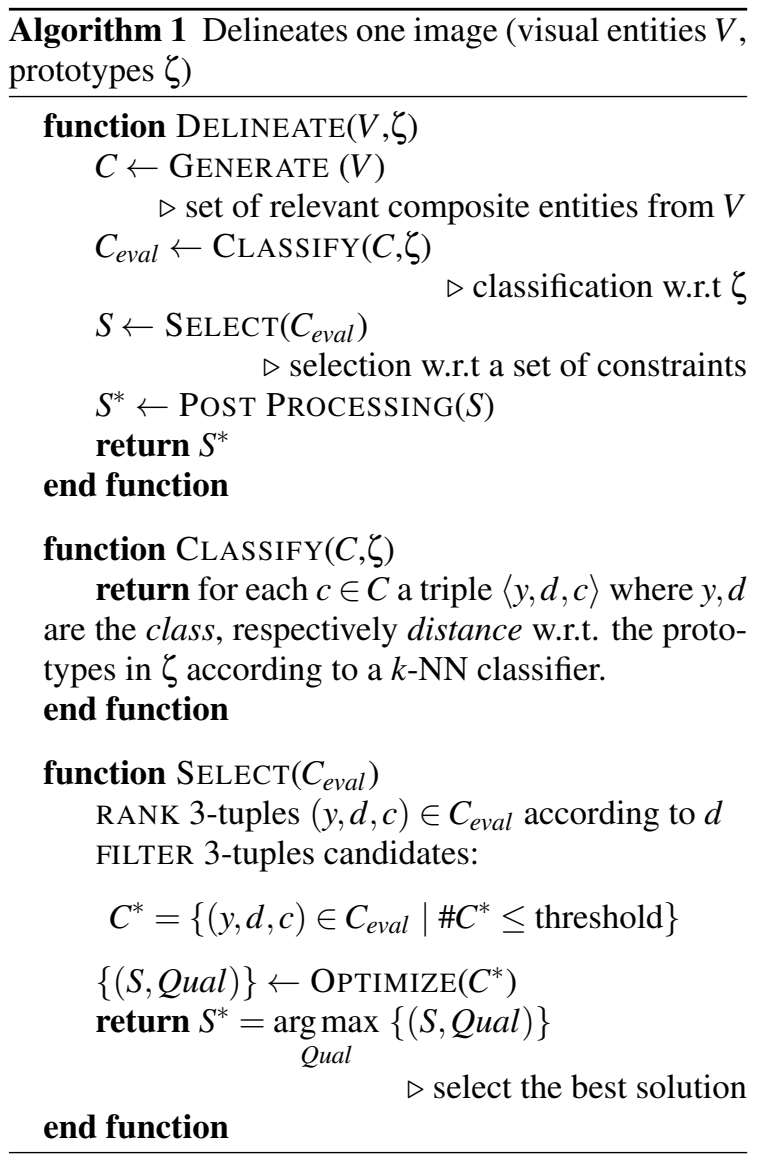

cally chosen maximum value. Each composite entity maps a local configuration of visual entities, induced by the close relation, which is thresholded on the image characteristics. To each of these subgraphs a composite entity $c$ is created and connected to all its visual entities using membership relations. The spatial relations are evaluated for pairs of visual entities, thereby forming locally connected subgraphs. The result is the subset $C$ of candidate composite entities.

The candidate generation is done recursively for every image. It starts with a less strict threshold on the close relation and it decreases the threshold at each iteration until the constraint on the upper bound of the size of $C$ is met. To find the best delineation in case of noisy information, composite entities representing a small number of visual entities are also needed. For example, if the image contains some parts of a (hypothetical) house, they can be regarded as configurations on their own (e.g. the partial house $i c_{3}$ in Figure 2(b)).

II Composite Entity Classification (CLASSIFY). At all layers, except the primitive, a $k$-nearest-neighbor approach based on a distance measure between two composite entities is used for composite entity classi- fication. Each composite entity is represented by its corresponding visual interpretation $I$. A matching between any two interpretations $I_{1}$ and $I_{2}$, is a mapping such that each entity in $I_{1}$ is mapped to at most one entity in $I_{2}$. In terms of the graph representation, this corresponds to mapping the vertices from $I_{1}$ to those of $I_{2}$ (Figure 4 ). The distance function $d\left(I_{1}, I_{2}\right)$ that we define measures the quality of the mapping and has two components. One characterizes the structure similarity, the other the appearance. Our choice is justified by the fact that both aspects may have impact on the matching score.

II-A Classification: Structure. To evaluate how well two logical interpretations match structurally, we must calculate their generalization (common part). We employ a recent result of (De Raedt and Ramon, 2009) on metrics. It targets the minimally general generalizations of two interpretations, but applies to different types of objects, including graphs. We choose the object identity (OI)-subsumption order (Ferilli et al., 2003), which, for graphs, corresponds to subgraph isomorphism. The minimally general generalization (mgg) then is the maximal common subgraph. This means that vertices in the subgraph can be mapped to at most one vertex in the supergraph, imposing an exact structure matching, and thus the mgg is not necessarily unique (De Raedt, 2008). Example 1 illustrates the mgg under OIsubsumption.

Example 1. Let $I_{1}=\left\{\operatorname{cRight}\left(\mathrm{o}_{1}, \mathrm{o}_{2}, 2\right)\right\}$ and $I_{2}=\left\{\operatorname{cRight}\left(o_{3}, o_{4}, 2\right), \operatorname{cRight}\left(o_{5}, o_{4}, 2\right)\right\}$.

Under OI-subsumption there are two possible mggs:

$\operatorname{mgg}_{O I}^{0}\left(I_{1}, I_{2}\right)=\left\{\operatorname{cRight}\left(\mathrm{X}_{1}, \mathrm{X}_{2}, 2\right)\right\}$

$\theta_{1}^{0}=\left\{\mathrm{X}_{1} / \mathrm{o}_{1}, \mathrm{X}_{2} / \mathrm{o}_{2}\right\}, \theta_{2}^{0}=\left\{\mathrm{X}_{1} / \mathrm{o}_{3}, \mathrm{X}_{2} / \mathrm{o}_{4}\right\}$

$\operatorname{mgg}_{O I}^{1}\left(I_{1}, I_{2}\right)=\left\{\operatorname{cRight}\left(\mathrm{X}_{1}, \mathrm{X}_{2}, 2\right)\right\}$

$\theta_{1}^{1}=\left\{\mathrm{X}_{1} / \mathrm{o}_{1}, \mathrm{X}_{2} / \mathrm{o}_{2}\right\}, \theta_{2}^{1}=\left\{\mathrm{X}_{1} / \mathrm{o}_{5}, \mathrm{X}_{2} / \mathrm{o}_{4}\right\}$.

with

Consequently, the mgg for two interpretations $I_{1}$ and $I_{2}$ results in the set $\operatorname{mgg}_{\text {all }}=\left\{\operatorname{mgg}\left(I_{1}, I_{2}\right)\right\}$. Using one $\mathrm{mgg}$ from the set, the distance between two interpretations $I_{1}$ and $I_{2}$ is equivalent to:

$$
d_{s}=\left|I_{1}\right|+\left|I_{2}\right|-2\left|\operatorname{mgg}\left(I_{1}, I_{2}\right)\right|
$$

where $|\cdot|$ is the number of the vertices in the interpretation. From this, it is straightforward to derive a normalized structural distance $d_{n s}\left(I_{1}, I_{2}\right)$. Similar distance measures are defined in (Nienhuys-Cheng, 1997; Horváth et al., 2001; Kirsten et al., 2000).

II-B Classification: Appearance. In addition to structural similarities, properties of entities (e.g., color) are important. If $m g g$ represents the maximal common structure between two interpretations $I_{1}$ and $I_{2}$, then $m g g \theta_{1}$ and $m g g \theta_{2}$ are specialized maximal common parts of $m g g$ that correspond to $I_{1}$ and $I_{2}$, respectively. The substitutions $\theta_{1}$ and $\theta_{2}$ specify the mapping between different entities. Indeed, if 
$V / e_{1} \in \theta_{1}$ and $V / e_{2} \in \theta_{2}$ then $e_{1}$ is mapped onto $e_{2}$. We can now define a normalized appearance distance between the two interpretations $I_{1}$ and $I_{2}$ as:

$$
d_{n a}\left(I_{1}, I_{2}\right)=\frac{1}{|m g g|} \times \sum_{a \in m g g} d_{0}\left(a \theta_{1}, a \theta_{2}\right),
$$

where $a$ is an atom in $m g g$. Since $m g g$ gives the common structure of the two interpretations, in order to compute $d_{n a}\left(I_{1}, I_{2}\right)$ we start from $m g g$ and specialize each atom $a \in m g g$, such that $a \theta_{1}$ and $a \theta_{2}$ are ground atoms with the same predicate symbol $a$. Let $S$ denote the set of all symbols, then the distance $d_{0}: S \times S \rightarrow[0,1]$ is a normalized distance measure defined for our particular application in the following way. Let $t_{i}, s_{i}$ be attributes, then:

$$
d_{0}\left(a\left(t_{1}, \ldots t_{n}\right), a\left(s_{1}, \ldots, s_{n}\right)\right)=\frac{1}{n} \times \sum_{i=1}^{n} d_{0}\left(t_{i}, s_{i}\right)
$$

For discrete attributes we employ the hamming distance $d_{0}\left(t_{1}, t_{2}\right)=1$ if $t_{1}=t_{2}$, otherwise 0 . For numerical attributes in the range [min, max]:

$$
d_{0}\left(t_{1}, t_{2}\right)=\frac{a b s\left(t_{1}-t_{2}\right)}{\max -\min }
$$

The structural and appearance-based aspects of the distance measure are combined into a single measure using a (normalized) weighted average:

$$
d_{s a}\left(I_{1}, I_{2}\right)=w_{s} \times d_{n s}\left(I_{1}, I_{2}\right)+w_{a} \times d_{n a}\left(I_{1}, I_{2}\right),
$$

where $w_{s}+w_{a}=1$. These weights can be supplied or learned. Because the mgg of interpretations $I_{1}$ and $I_{2}$ is not unique, the global normalized distance between $I_{1}$ and $I_{2}$ finally is:

$$
d\left(I_{1}, I_{2}\right)=\min _{m \in \operatorname{mgg}_{\text {all }}} d_{s a}\left(I_{1}, I_{2}\right) .
$$

Next, we employ a k-nearest neighbor classifier (KNN in function CLASSIFY). Given the set of composite entities $C$ and the set of prototypes $\zeta$, the algorithm evaluates the quality of each composite entity by computing the distance to the prototypes and classifies it based on the majority vote of its neighbors. The algorithm returns the set $C_{\text {eval }}$ of 3-tuples $\left(y, d_{\zeta}, c\right)$, where $y$ is the class of $c \in C$ and $d_{\zeta}$ is the mean distance from $c$ to the elements of the subset $\zeta^{y} \subseteq \zeta$ describing only concepts of class $y$.

III Composite Entity Selection (SELECT). In the function SELECT we first rank the set of composite entities of interest $C$ according to their distances to the nearest prototypes in $\zeta$, and use a threshold on the number of candidates to select the best set $C^{*}$. This step is optional, but recommended as a large space of composite entities $C$ is usually generated. From this reduced set, we then want to select those that together explain best (most of) the visual features at that layer. To this end, we formulate the composite entity selection problem as a maximum weighted independence set problem.

Let $G=(V, E, W)$ be an undirected graph, where $V, E$ and $W$ are the set of vertices and edges and a vertex weighting function, respectively. An independent set is a set $S \subseteq V$ such that $\forall e \in E$ the two end vertices of $e$ do not belong to $S$ simultaneously. A maximum weighted independence set problem (WISP) is formulated as follows: given an input graph $G=(V, E, W)$, find the independence set $S$ of vertices in $V$ such that the value $W(S)$ is subject to maximization. In order to convert our problem to a WISP problem we have to find the correspondence to the input graph $G=(V, E, W)$ and the independence set $S$. In our case:

- $V$ becomes the set of composite entities $C^{*}$.

- we use the set of edges $E$ to model constraints between composite entities, that is the solution must contain only composite entities that do not share any visual entities. This constraint is considered through the independence property itself by inserting an edge between any two $C$ which share at least one visual entity:

$$
E=\left\{e\left(c_{1}, c_{2}\right) \mid c_{1}, c_{2} \in C^{*}, V\left(c_{1}\right) \cap V\left(c_{2}\right) \neq \varnothing\right\}
$$

- the vertex weighting function $W: V \rightarrow \mathbb{N}$ is

$$
W_{c}=\sigma\left(1-d_{\zeta}(c, \zeta)\right), \forall c \in C^{*}
$$

where $\sigma$ is a function which proportionally amplifies higher scores to ensure the selection of best scored composite entities. The function that we want to maximize is then $W(S)=\sum_{c \in S} W_{c}$, where $S$ is one independence set solution.

The solution to the WISP problem is given by the function OPTIMIZE. This is known to be a NP-hard optimization problem and both exact and approximation algorithms exist (Busygin, 2006). For the exact case we use a branch-and-bound algorithm for the maximum clique problem, which is computationally equivalent to the maximum independent set problem computed on the complement graph (see (Östergård, 2002) for more details). We therefore replace our OPTIMIZE function with the Cliquer optimizer ${ }^{1}$. For the approximation case we use the algorithm for the maximum weight clique problem proposed in (Busygin, 2006). We employ the QUALEX-MS optimizer ${ }^{2}$ instead of our OPTIMIZE function. Other approximation methods are also known to work in polynomial

\footnotetext{
${ }^{1}$ Available at http://users.tkk.fi/pat/cliquer. html.

${ }^{2}$ Available at http://www. stasbusygin.org.
} 
time (Lozin and Milanic, 2010). However these are adequate for particular (i.e., planar) graphs, while our selection problem deals with general graphs. If the size of $C^{*}$ is in a certain range we use the exact optimizer, otherwise the approximate one. This gives acceptable results in practice.

The algorithm follows the same principle for all layers of the hierarchy. However there are differences at each layer with respect to i) the interpretations generated (both size and structure) and therefore the composite entities and ii) the distance function which is tuned for each layer.

IV (Post Processing) We employ two steps.

IV-A Bounding Box Prediction. The end goal of our framework is to predict the bounding boxes of detected objects. We use the subgraph $V S_{c}$ of the composite entity to predict a bounding box for the object $c$. This is implemented by mapping the bounding boxes of the visual entities in $V_{c}$ (i.e., vectors of $2 \mathrm{D}$ locations) to the corners of the object bounding box.

IV-B Non-Maximum Suppression (NMS). Either the KNN classifier or the selection step may give multiple spatially overlapping detections for each instance of an object. The selection step ensures that detections do not share any visual entities, however their bounding boxes can still overlap. We use a greedy procedure for eliminating repeated detections via non-maximum suppression. After applying the bounding box prediction described above we have a set of detections for an object category in an image. Each detection is defined by a bounding box and a score. We sort the detections by score, and greedily select the ones with highest score, while suppressing detections with bounding boxes that are more than some percentage (which varies depending on the setting) covered by a bounding box of a previously selected detection. A similar NMS step is taken in (Felzenszwalb et al., 2010).

\section{APPLICATION AND EXPERIMENTAL EVALUATION}

\section{Dataset and Application}

We first describe the application of our method to 2D street view images (Figure 5). These commonly display a rich structure (and variety), yet are often quite consistent in terms of structure in a row of houses. We have annotated ${ }^{3} 60$ images of rows of house facades from different countries. A number of

\footnotetext{
${ }^{3}$ Using the LABELME toolbox (Russell et al., 2008).
}
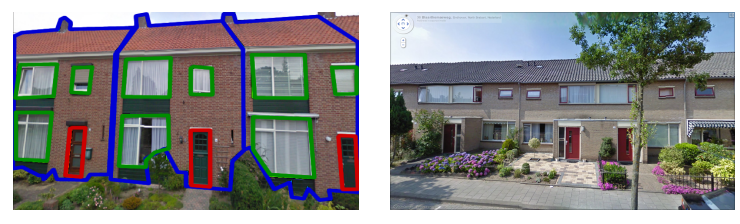

Figure 5: Images of houses in Eindhoven; an annotated training image is on the left; a testing image is on the right.

20 images were collected by ourselves, the rest from Google Street View. All images show near-frontal views of the houses and no further rectification was performed. Each image has a resolution of $600 \times 800$ pixels. On these images, windows, doors and houses were manually annotated. We use the close to the right (cRight), close above (cAbove) and touch to the right (tRight) spatial relations as illustrated in Example 2. An Euclidian distance threshold is used for the close relation defined relatively to the size of the objects. The background knowledge can easily be extended with new relations, to enable even richer relational representations of visual data.

Example 2. The background knowledge for the spatial relation cRight:

closeto $(A, B$, Dist $) \leftarrow b b\left(A, B B_{1}\right), b b\left(B, B B_{2}\right), A \neq B$,

distance $\left(B B_{1}, B B_{2}\right.$, Dist $)$, Dist $<$ threshold.

$c \operatorname{Right}(A, B, D i s t) \leftarrow b b\left(A, B B_{1}\right), b b\left(B, B B_{2}\right)$,

right $\left(B B_{1}, B B_{2}\right)$, closeto $(A, B$, Dist $)$.

where $b b$ is the bounding box of a visual entity.

We make use of three layers in a four-level hierarchy: primitive, object and house layers (Figure 6).

Primitive Layer: This layer takes as input image pixels and groups them in corner-like features with local descriptors. We employ the KAS feature detector (Ferrari et al., 2008) to detect interest points formed by chains of $k$ connected, roughly straight contour segments. We use $k=2$ to detect corner-like shapes and solve the classification problem by attaching a category label from the set $Y=\{c$ Type $00, c$ Type 01, cType $10, c$ Type 11$\}$ to each corner-like candidate. These labels represent topright, top-left, bottom-right and bottom-left corners and are established based on the orientation of the segments composing the $2 \mathrm{AS}$ feature. The selection is done in two steps. Firstly, we only keep squarelike corners with an angle $(90-\delta)^{\circ}<\alpha<(90+$ $\delta)^{\circ}$. Secondly, we describe the selected 2 AS features with HOG descriptors (Dalal and Triggs, 2005) and train a binary classifier on these descriptors to discard irrelevant corner features found on other structures than buildings (e.g., vegetation or cars). Object layer annotations represent training data for this step. Object Layer: Visual entities at this level are sparse detected corner features. Each corner has a lo- 
cal HOG descriptor ${ }^{4}$ as an attribute on which an appearance-based distance component can be computed. We solve this by using first a trained a classifier to map the attribute to either a window or door label, and then computing a discrete distance between these labels. An additional attribute is the corner type (e.g., cType00). Based on our spatial theory, attributes representing the Euclidean distance between bounding boxes of spatially related entities, contribute also to the appearance-based distance. Composite entities represent possible doors or windows and are defined by subgraphs consisting of 3 up to 4 visual entities.

House Layer: Visual entities here are doors and windows found at the object layer, and composite entities represent possible houses. Again we employ our spatial theory to find potential composite entities, and derive attributes for the spatial relations between visual entities. Attributes of visual entities at this layer are the labels door and window. Composite entities are defined by subgraphs consisting of 2 up to 6 visual entities, estimated from the training data.

\section{Experimental Evaluation}

The experiments were performed in two different phases. In a first phase, we performed experiments at single layers independently. More precisely, we used as input for the learning task at one single layer the annotated (or segmented, for the object layer) training data at that layer and then employed our method to compute the output. In this way, it is possible to get an appreciation of how difficult the learning problem is and what are the limitations of the data at each layer. In a second phase, we performed experiments in the full hierarchical setting, that is, the inputs are image pixels and the outputs are at the house layer. This allows us to estimate how good the hierarchical approach works.

Because we deal with a detection problem we adopt the evaluation measures used in information retrieval. We measure performance in terms of the number of true and false detections in a test dataset. In our setting the positives are all the composite entities selected via the selection function. We evaluate the performance using the overlap measure, which is also the PASCAL VOC (Everingham et al., 2008) criterion. We compare the bounding box $B B_{d}$ corresponding to the detected concept to the ground-truth bounding box $B B_{t}$ in manually annotated data. If area $\left(B B_{d} \cap B B_{t}\right) / \operatorname{area}\left(B B_{d} \cup B B_{t}\right)>0.5$, then $B B_{d}$ is a true positive (TP), otherwise it is a false positive

\footnotetext{
${ }^{4} \mathrm{~A}$ variation of the HOG descriptor with 16 orientation bins instead of 9 showed improved results. We used a window size of $128 \times 128$ pixels and a block size of $8 \times 8$ cells.
}

(FP). The precision $P$ is TP divided by the total number of predicted components. The recall $R$ is TP divided by the number of ground-truth components in the test set. The Fl score is a measure of accuracy and is the harmonic mean of precision and recall.

The problem of detection is often posed as a classification task, namely distinguishing in the image the class of interest with some score. Such a classifier can be turned into a detector by sliding it across the image and thresholding the scores of the hypothesis to obtain a precision-recall curve. Differently, our formulation builds on top of a kNN classifier by selecting interesting (already scored) candidates which together find the best semantic segmentation of the image. Since they are together part of the solution, they are all predicted positive instances (except the spatially overlapping ones solved by the final NMS step). As a result, there is no obvious threshold that can be varied to trade-off precision vs. recall and instead of a precision-recall curve, the performance is measured as a precision-recall point. Since we are interested to measure the impact of structure on our detection problem, we vary the parameter $w_{s}$ of our model and show its influence on precision and recall values.

We have as parameters $k$ (in the $\mathrm{KNN}$ ) and the relative weights $w_{s}$ and $w_{a}$ (structure vs. appearance for classification). We experiment with different values of $k$ to evaluate the influence of the structure parameter $w_{s}$ on precision/recall values ${ }^{5}$.

\section{Results}

We have tested different values of $w_{s}$ at single layers independently and with the full hierarchy.

Experiment 1. Single layer segmentation - houses. At the house layer, we first test our approach directly on the ground-truth annotations of the underlying layer, that is, objects such as windows and doors. We vary $w_{s}$ from 0 to 1 to plot recall and precision in Figure 7. We stress that $w_{s}$ is not a threshold to trade precision for recall, but we use it to show the influence of using structure on the performance. We observe that if $k$ is large enough $(k \geq 30)$, the increase of the amount of structure increases precision/recall values. Indeed, in our setup the use of structure is essential to obtain good results. We notice that the approach is not very sensitive to a precise value of $w_{s}$ when $w_{s}>0.1$. For $k=60$ we obtain optimal values $\mathrm{R}=0.86, \mathrm{P}=0.83$; for $k=90, \mathrm{R}=0.92$ and $\mathrm{P}=0.9$; for $k=$ all, $\mathrm{R}=0.88$ and $\mathrm{P}=0.85$. They are all obtained when $w_{s}=0.4$ and we observe that the appearance component $w_{a}=0.6$ has also influence on obtaining optimal values of precision and recall. We also note

\footnotetext{
${ }^{5}$ We choose $w_{s}$ as the free parameter; $w_{a}=1-w_{s}$.
} 


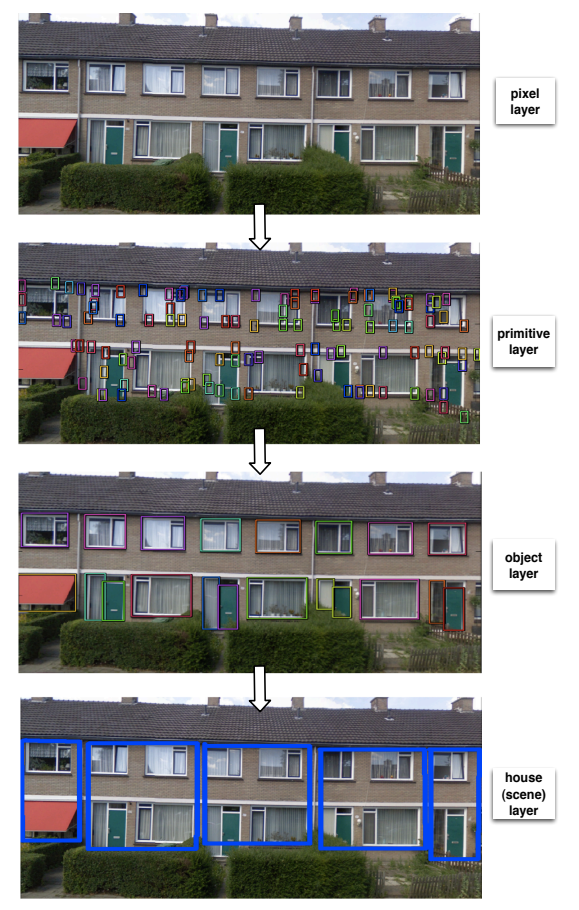

Figure 6: Data flow in the four-level hierarchy of the facades domain. Input layers: pixels, corner primitives and object entities. Corresponding output layers: corner primitives, object entities and house entities, respectively.

that, due to the selection procedure, precision and recall are highly coupled. For small values of $k$ recall and precision are much lower for any $w_{s}$. Given the structural variability at the house layer, a comparison with enough prototypes is needed.

Experiment 2. Single layer segmentation - objects. At the object layer the experiments are performed directly with available detected KAS from the primitive layer (not with annotations). They show that the variation of the structure still has an influence, though it is more limited. This can be explained by the fact that windows and doors have mostly the same structure. However, at the object layer the structure still has an indirect influence, as it is needed for computing appearance-based aspects. We ran experiments with different values for $k$ and $w_{s}$. The results are shown in Figures 8 and 9 for classes door and window, respectively. The maximal values $\mathrm{R}=0.42, \mathrm{P}=0.47$ for class door and $\mathrm{R}=0.61, \mathrm{P}=0.35$ for class window are obtained for parameters $k=50, w_{s}=0.3, w_{a}=0.7$ and $k=50, w_{s}=0.5, w_{a}=0.5$, respectively. However, results for $k=10, k=75$ and $k=150$ are also close. A NMS step with $50 \%$ overlap was applied after the selection phase and before the evaluation.

Experiment 3. Hierarchical segmentation houses. We evaluate detection results at the house layer using the full hierarchy. From the raw image we
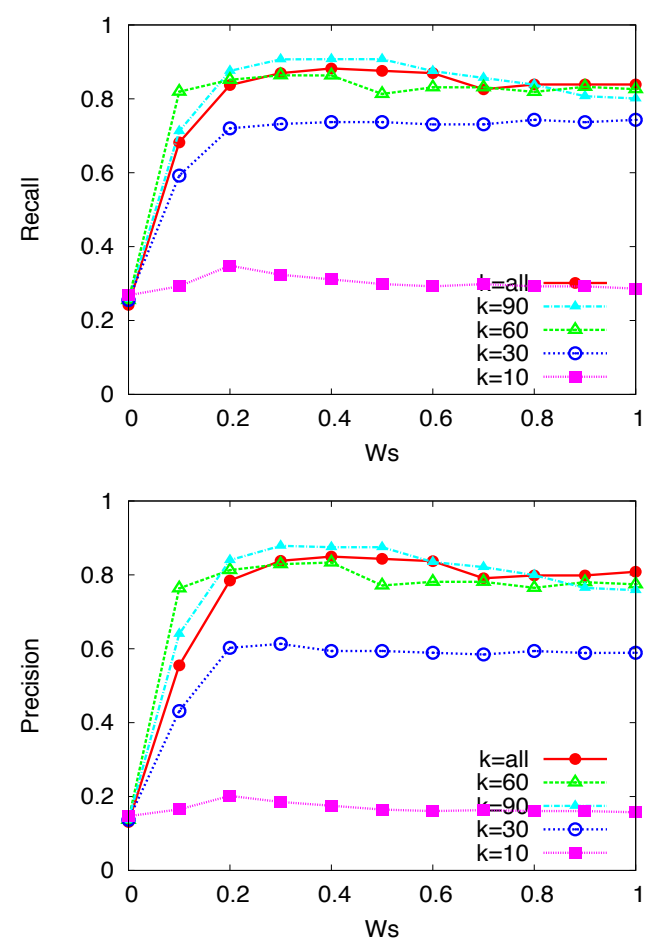

Figure 7: House layer segmentation (annotations). The influence of structure on precision/recall for different $k$.

first detect the KAS primitives. These are then employed further as input to detect windows and doors. At this point there are 2 possible ways to proceed. We can select relevant windows and doors (via the described selection step) at the object layer and use this result as input for the house layer. However, this gives less good results $(\mathrm{R}=0.32$ and $\mathrm{P}=0.5)$, as a high enough recall is required from the object layer to obtain rich enough visual interpretations of images.

Alternatively, instead of the full selection step, we consider the top ranked composite entities. In this way, the full selection is replaced by a less selective mechanism, improving recall at the object layer. The selected candidates become visual entities at the house layer. This improves the results, as shown in Figure 10, to obtain for $k=90, \mathrm{R}=0.6$ and $\mathrm{P}=0.63$, while for $k=a l l, \mathrm{R}=0.61$ and $\mathrm{P}=0.65$. The optimal results are obtained when $w_{s}=0.4$.

In all experiments we perform a 5 -fold cross validation on the dataset with the same folds. The implementation combines code written in Prolog, Matlab and $\mathrm{C}$. We are able to delineate houses and to separate them from neighboring houses, even when attached. Some qualitative results are presented in Figure 1214. The higher we get in the hierarchy and therefore richer in the semantics, the more relevant the structural aspect becomes. 

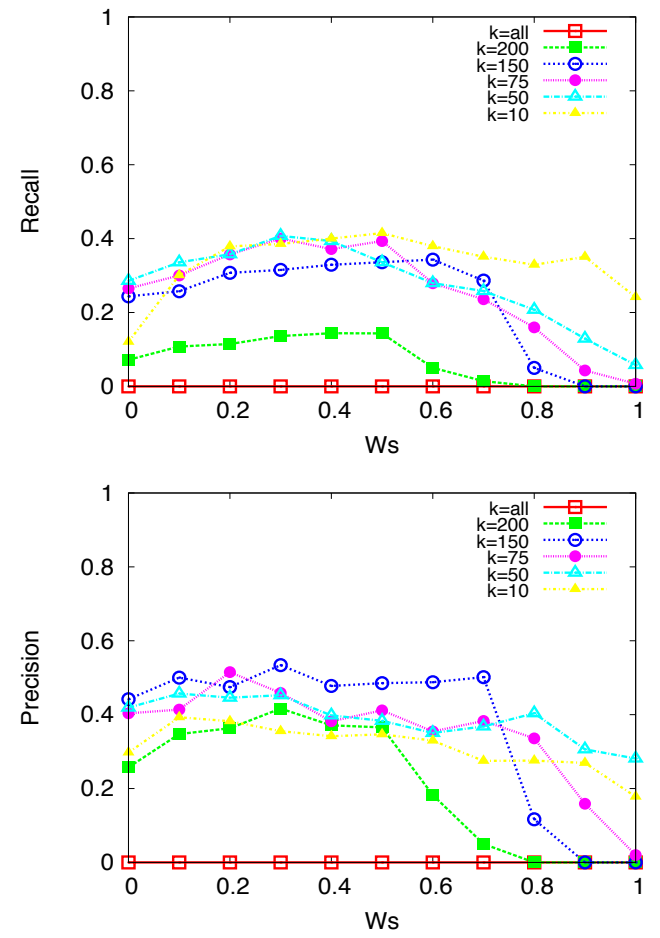

Figure 8: Object layer segmentation for class door. The influence of structure on precision/recall for different $k$.

Comparison to baselines: The goal of this work is not so much to compete with powerful detectors, often building on dense representations from the literature, but rather to evaluate how structure can be flexibly exploited in detection problems in general. We show that even when starting from relatively sparse and simple cues (Figure 6, primitive layer), detection and delineation of complex objects is feasible, thanks to the use of structure. Moreover, rather than just detecting bounding boxes of objects, our method returns a full semantic hierarchical interpretation of the scene, decomposing each object into its constituents parts. Nevertheless, for reference, we compare our method with several baseline detectors to assess the difficulty of the dataset.

Baseline 1. Objectness. As a first baseline, we use the object detector proposed in (Deselaers and Ferrari, 2010) and employ the objectness measure to quantify how likely it is for an image window (i.e., patch) to contain an object. The measure combines several image cues, such as: multi-scale saliency, color contrast, edge density and straddleness. Its main purpose is to predict, given an image, location priors in the form of image windows to boost class specific object detectors. The window sampling is done according to the objectness distribution in the image. We use it
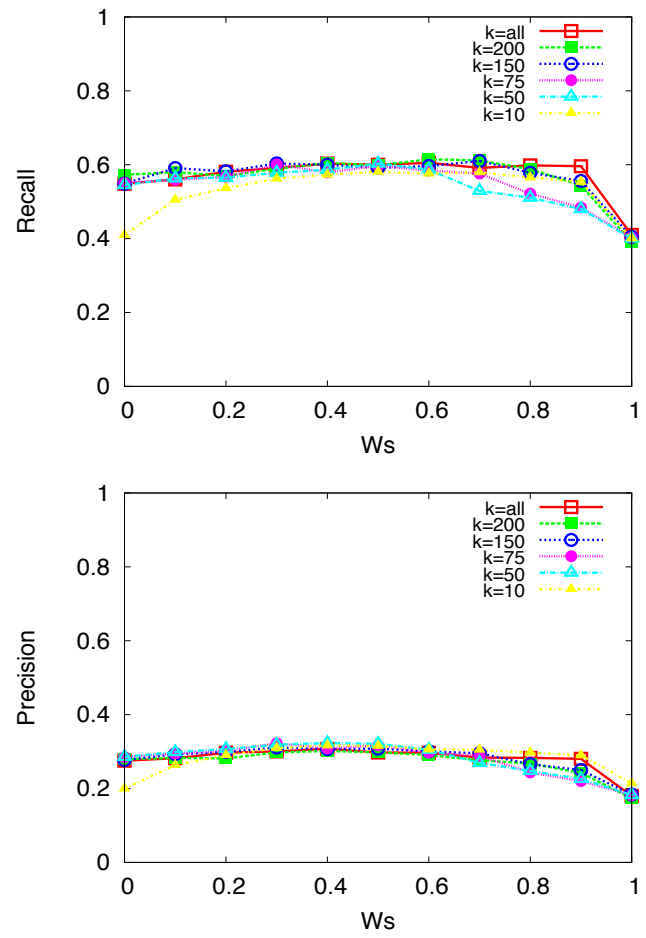

Figure 9: Object layer segmentation for class window. The influence of structure on precision/recall for different $k$.

here as a first baseline house detector ${ }^{6}$. We run the detector with 100 window samples ${ }^{7}$ and then employ the PASCAL VOC (Everingham et al., 2008) overlapping criterion to establish the correct label of each sample (i.e., house or none).

Baseline 2. Objectness + HOG. As a second baseline we combine the objectness measure with a separate classifier trained $^{8}$ for the class house on HOG feature descriptors (Dalal and Triggs, 2005). The objectness classifier is used as a prior distribution to sample relevant hypotheses in the image, while the HOG classifier is used to re-score them. We first sample 100 house candidates in each image and then employ the specialized classifier to improve the predictions.

Baseline 3. Template Matching with Boosting. As a third baseline we use the boosting approach ${ }^{9}$ in (Torralba et al., 2004) to train an ensemble of weak detectors for the class house. Each weak detector computes

\footnotetext{
${ }^{6}$ Version 1.5, available at http://www.vision.ee. ethz.ch/ calvin/software.html.

${ }^{7}$ According to the detector specifications. Increasing or decreasing the number of samples did not improve results.

${ }^{8}$ Using the LIBSVM library available at: http: / / www . csie.ntu.edu.tw/ cjlin/libsvm/.

${ }^{9}$ Available at http://people.csail.mit.edu/ torralba/shortCourseRLOC/boosting/boosting. html.
} 

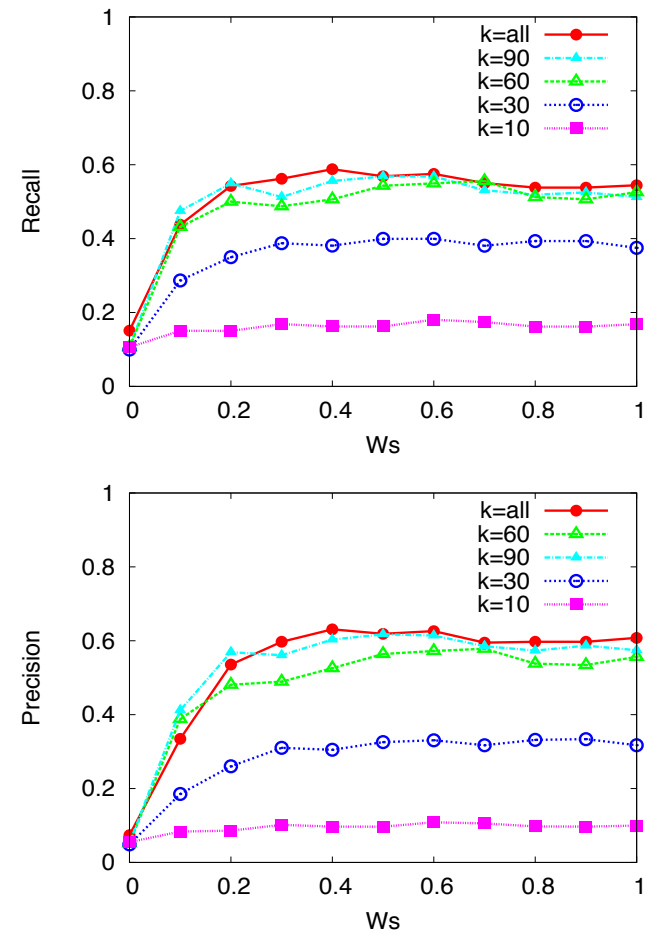

Figure 10: Hierarchical segmentation. The influence of structure on precision/recall for different $k$.

\begin{tabular}{cccc}
\hline Method & Recall & Precision & $F 1$ \\
\hline Objectness & 0.14 & 0.1 & 0.12 \\
Objectness + HOG & 0.23 & 0.11 & 0.14 \\
Feature boosting (120) & 0.57 & 0.48 & 0.52 \\
kNN classifier & 0.4 & 0.3 & 0.35 \\
Our method & $\mathbf{0 . 6 1}$ & $\mathbf{0 . 6 5}$ & $\mathbf{0 . 6 3}$ \\
\hline
\end{tabular}

Table 1: Hierarchical segmentation and comparison to baselines; house layer.

template matching with a localized patch in object centered coordinates. The features are obtained using a convolution mask tailored to the normalized correlation between the search patch and the template. Individual houses can be more effectively detected using a template matching approach than a texture-based one, since houses in the same row have the same texture and most street scenes greatly vary in texture across the dataset. In our experiments we use different numbers of weak classifiers (see Figure 11).

Table 1 shows the results for all baselines and our method. The F1 values showed are the maximum F1 scores over all precision-recall points in the precision-recall curves as in Figure 11. We also include results of the intermediary classification step to show the benefits of the selection function. Although the baseline detectors perform reasonably well for the

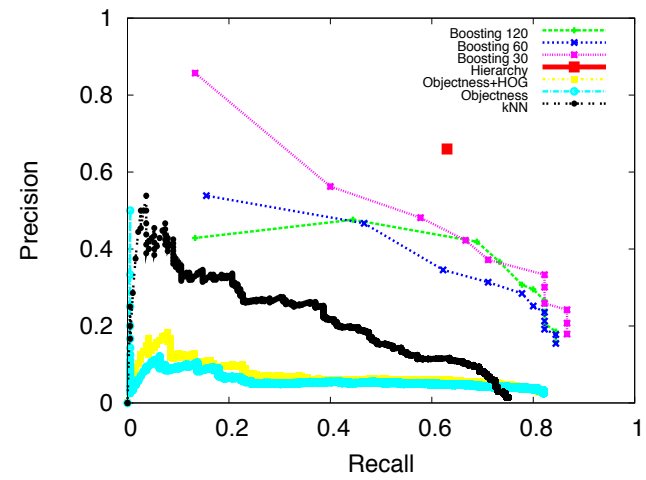

Figure 11: Baseline precision-recall curves; class house.

house detection problem, none of these detectors incorporates a fine-grained decomposition of a house, in the form of structured output which explains the image in the same way as our framework. In addition, our method still outperforms the baselines although we start from sparse features and therefore, a less rich appearance-based component. We only use as features the corners estimated from 2AS and a HOG descriptor on its (reduced) neighborhood. This is opposed to the employed baselines which are optimized for dense cues and a richer appearance component.

To summarize, we show that our framework gives promising results for the difficult detection tasks at both individual levels and through the full hierarchy. A challenging aspect is the propagation of candidates up through the hierarchy. The recall of structures needed at a higher level can limit good performance at that layer. If the number of allowed candidates is high enough - which means that we do not just propagate the sole best solution, but a larger number of candidate solutions - we enable the higher layer to select from more candidates and achieve better performance. This balance between generating many candidates and propagating a suitable number of candidates must be determined by the domain at hand, and an interesting research question is how to computationally deal with this trade-off. Overall, our results clearly show the feasibility and effectiveness of our approach by combining relational knowledge representations with computational vision.

\section{Conclusions and Future Work}

We have presented a novel general framework for hierarchical image understanding, incorporating distance-based classifications, relational, spatial knowledge representation and robust visual feature recognition. The experiments show i) the interplay 
between structural and appearance-based aspects in the recognition task and ii) good detection results both at single layers and full hierarchy. This work explores a new relational scheme for solving computer vision tasks and we believe that there is still room for improvement. Three strong points of the approach are that i) we do not assume availability of a full model of the domain (e.g., a grammar) but only a set of annotated examples, which is more natural and easier to obtain, ii) the framework can easily be extended by adding relational/spatial background knowledge, or replacing the classifiers by other similarity functions or kernels and iii) the approach incorporates a fine-grained decomposition of a house in the form of structured output which explains the image, as opposed to existing detectors.

In future work, we will extend the framework and employ it for other recognition tasks (e.g., people detection), suitable online annotated data, as well as including denser cues as appearance features. We suspect that extending the distance computations with kernel-based learning for structured data (e.g., relational representations) will boost recognition performance. Another interesting direction is to provide more contextual knowledge in the recognition, for example to take higher-levels into account directly when classifying structures at lower levels as top-down feedback. A general goal is to explore the boundaries between robust computer vision, highlevel knowledge representation and machine learning.

\section{Acknowledgments}

The first author thanks Gianluigi Greco, Bernd Gutmann, Siegfried Nijssen and Paulo Moura for their valuable comments, discussions, advice and support.

\section{REFERENCES}

Bar-Hillel, A. and Weinshall, D. (2008). Efficient learning of relational object class models. IJCV, 77(1-3):175198.

Busygin, S. (2006). A new trust region technique for the maximum weight clique problem. Discrete Appl. Math., 154(15):2080-2096.

Caetano, T. S., McAuley, J. J., Cheng, L., Le, Q. V., and Smola, A. J. (2009). Learning graph matching. TPAMI, 31(6):1048-1058.

Dalal, N. and Triggs, B. (2005). Histograms of oriented gradients for human detection. In CVPR, pages 886893.
De Raedt, L. (2008). Logical and Relational Learning. Springer.

De Raedt, L. and Ramon, J. (2009). Deriving distance metrics from generality relations. Pattern Recognition Letters, 30(3):187-191.

Deselaers, T. and Ferrari, V. (2010). Global and efficient self-similarity for object classification and detection. In $C V P R$, pages $1633-1640$.

Dubba, K. S. R., Cohn, A. G., and Hogg, D. C. (2010). Event model learning from complex videos using ILP. In ECAI, pages 93-98.

Esposito, F., Malerba, D., and Semeraro, G. (1992). Classification in noisy environments using a distance measure between structural symbolic descriptions. PAMI, 14(3):390-402

Everingham, M., Van Gool, L., Williams, C. K. I., Winn, J., and Zisserman, A. (2008). The PASCAL Visual Object Classes Challenge 2008.

Felzenszwalb, P., Girshick, R., McAllester, D., and Ramanan, D. (2010). Object detection with discriminatively trained part-based models. TPAMI, 32(9):1627 -1645 .

Fergus, R., Perona, P., and Zisserman, A. (2007). Weakly supervised scale-invariant learning of models for visual recognition. IJCV, 71(3):273-303.

Ferilli, S., Mauro, N. D., Basile, T. M. A., and Esposito, F. (2003). A complete subsumption algorithm. In $A I^{*} I A$ 2003, pages 23-26.

Ferrari, V., Fevrier, L., Jurie, F., , and Schmid, C. (2008). Groups of adjacent contour segments for object detection. TPAMI, pages 36-51.

Getoor, L., Koller, D., Taskar, B., and Friedman, N. (2000). Learning probabilistic relational models with structural uncertainty. In Proceedings of the ICML-2000 Workshop on Attribute-Value and Relational Learning: Crossing the Boundaries, pages 13-20.

Hanson, A. and Riseman, E. (1978). Visions: A computer system for interpreting scenes. In CVS78, pages $303-$ 333.

Harchaoui, Z. and Bach, F. (2007). Image classification with segmentation graph kernels. In $C V P R$, pages $1-$ 8 .

Hartz, J. (2009). Learning probabilistic structure graphs for classification and detection of object structures. In ICMLA' 09 , pages 5-11.

Hartz, J. and Neumann, B. (2007). Learning a knowledge base of ontological concepts for high-level scene interpretation. In ICMLA, pages 436-443.

Horváth, T., Wrobel, S., and Bohnebeck, U. (2001). Relational instance-based learning with lists and terms. $M L, 43(1 / 2): 53-80$.

Kirsten, M., Wrobel, S., and Horváth, T. (2000). Distance based approaches to relational learning and clustering. Relational Data Mining, pages 213-230. 
Koutsourakis, P., Simon, L., Teboul, O., Tziritas, G., and Paragios, N. (2009). Single view reconstruction using shape grammars for urban environments. In $I C C V$, pages 1795-1802.

Li, L.-J., Socher, R., and Fei-Fei, L. (2009). Towards total scene understanding: Classification, annotation and segmentation in an automatic framework. CVPR, 0:2036-2043.

Lippow, M. A., Kaelbling, L. P., and Lozano-Perez, T. (2008). Learning grammatical models for object recognition. In Technical Report.

Lozin, V. and Milanic, M. (2010). On the maximum independent set problem in subclasses of planar graphs. Journal of Graph Algorithms and Applications, 14:269-286.

Muggleton, S. and Buntine, W. L. (1988). Machine invention of first order predicates by inverting resolution. In $M L$, pages 339-352.

Müller, P., Zeng, G., Wonka, P., and Van Gool, L. J. (2007). Image-based procedural modeling of facades. ACM Transactions on Graphics, 26(3):85.

Nienhuys-Cheng, S.-H. (1997). Distance between herbrand interpretations: A measure for approximations to a target concept. In $I L P$, pages 213-226.

Östergård, P. R. J. (2002). A fast algorithm for the maximum clique problem. Discrete Appl. Math., 120:197207.

Petrou, M. (2008). The tower of knowledge: a novel architecture for organising knowledge combining logic and probability. In Logic and Probability for Scene Interpretation, Dagstuhl Seminar Proceedings.

Pinz, A. J., Bischof, H., Kropatsch, W. G., Schweighofer, G., Haxhimusa, Y., Opelt, A., and Ion, A. (2009). Representations for cognitive vision: A review of appearance-based, spatio-temporal, and graph-based approaches. Electronic Letters on Computer Vision and Image Analysis, 7(2):35-61.

Russell, B. C., Torralba, A., Murphy, K. P., and Freeman, W. T. (2008). LabelMe: A database and web-based tool for image annotation. IJCV, 77(1-3):157-173.

Sudderth, E. B., Torralba, A., Freeman, W. T., and Willsky, A. S. (2008). Describing visual scenes using transformed objects and parts. IJCV, 77(1-3):291-330.

Szeliski, R. (2010). Computer Vision: Algorithms and Applications. Springer.

Torralba, A., Murphy, K. P., and Freeman, W. T. (2004). Sharing features: Efficient boosting procedures for multiclass object detection. In CVPR, pages 762-769.

Tuytelaars, T. and Mikolajczyk, K. (2007). Local invariant feature detectors: A survey. Foundations and Trends in Computer Graphics and Vision, 3(3):177-280.

Zhao, P., Fang, T., Xiao, J., Zhang, H., Zhao, Q., and Quan, L. (2010). Rectilinear parsing of architecture in urban environment. In CVPR, pages 342-349.

Zhu, S.-C. and Mumford, D. (2006). A stochastic grammar of images. Found. Trends. Comput. Graph. Vis., 2(4):259-362.

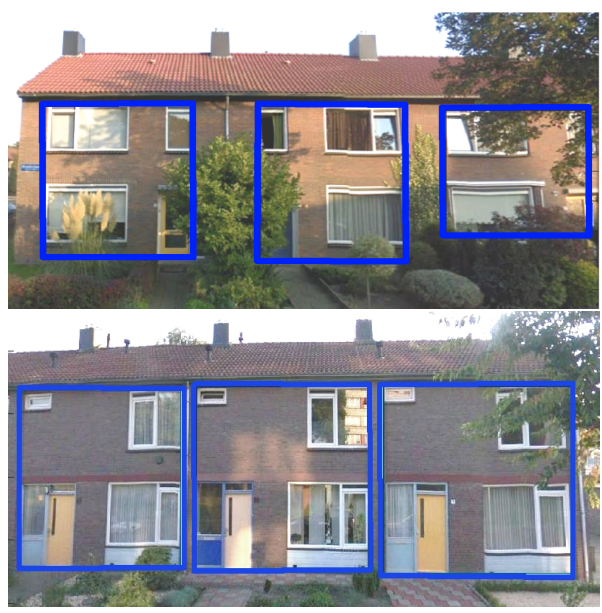

Figure 12: Segmentation of images with partial occlusions at single (house) layer.
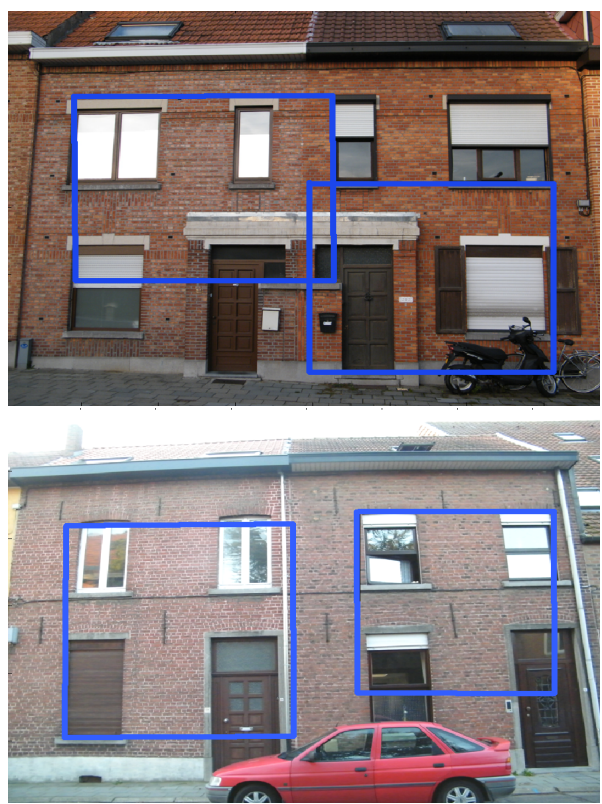

Figure 13: Segmentation of images with partial occlusions at the house layer using the hierarchy.

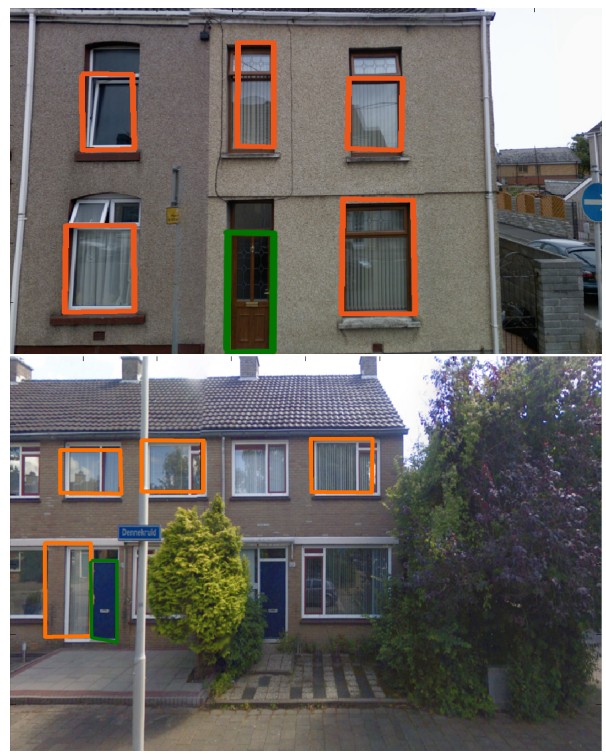

Figure 14: Segmentation of images at single (object) layer. Door detections are marked in green. 